\title{
Features and Agreement
}

\author{
Sam Bayer and Mark Johnson* \\ Cognitive and Linguistic Sciences, Box 1978 \\ Brown University \\ $\{$ bayer,mj\}@cog.brown.edu
}

\begin{abstract}
This paper compares the consistencybased account of agreement phenomena in 'unification-based' grammars with an implication-based account based on a simple feature extension to Lambek Categorial Grammar (LCG). We show that the LCG treatment accounts for constructions that have been recognized as problematic for 'unification-based' treatments.
\end{abstract}

\section{Introduction}

This paper contrasts the treatment of agreement phenomena in standard complex feature structure or 'unification-based' grammars such as HPSG (Pollard and Sag, 1994) with that of perhaps the simplest possible feature extension to Lambek Categorial Grammar (LCG) (Lambek, 1958). We identify a number of situations where the two accounts make different predictions, and find that generally the LCG account is superior. In the process we provide analyses for a number of constructions that have been recognized as problematic for 'unification-based' accounts of agreements (Zaenen and Karttunen, 1984; Pullum and Zwicky, 1986; Ingria, 1990). Our account builds on the analysis of coordination in applicative categorial grammar in Bayer (1994) and the treatment of Boolean connectives in LCG provided by Morrill (1992). Our analysis is similiar to that proposed by Mineur (1993), but differs both in its application and details.

The rest of the paper is structured as follows. The next section describes the version of LCG we use in this paper; for reasons of space we assume familiarity with the treatment of agreement in 'unificationbased' grammars, see Shieber (1986) and Pollard and Sag (1994) for details. Then each of the follow-

\footnotetext{
"We would like to thank Bob Carpenter, Pauline Jacobson, John Maxwell, Glynn Morrill and audiences at Brown University, the University of Pennsylvania and the Universität Stuttgart for helpful comments on this work. Naturally all errors remain our own.
}

ing sections up to the conclusion discusses an important difference between the two approaches.

\section{Features in Lambek Categorial Grammar}

In LCG semantic interpretation and long distance dependencies are handled independently of the feature system, so agreement phenomena seem to be the major application of a feature system for LCG. Since only a finite number of feature distinctions need to be made in all the cases of agreement we know of, we posit only a very simple feature system here. Roughly speaking, features will be treated as atomic propositions (we have no need to separate them into attributes and values), and a simple category will be a Boolean combination of such atomic 'features' (since we have no reason to posit a recursive feature structures either). In fact we are agnostic as to whether more complex feature systems for LCG are linguistically justified; in any event Dorre et. al. (1994) show how a full attribute-value feature structure system having the properties described here can be incorporated into LCG.

Following the standard formulation of LCG, we regard the standard LCG connectives ' $/$ ' and ' $Y$ ' as directed implications, so we construct our system so that $\alpha / \beta \beta^{\prime}$ can combine to form $\alpha$ if $\beta^{\prime}$ is logically stronger than $\beta$.

Formally, we adopt Morrill's treatment (Morrill, $1992)$ of the (semantically impotent) Boolean connectives ' $\wedge$ ' and ' $v$ ' (Morrill named these ' $\Pi$ ' and ' $u$ ' respectively). Given a set of atomic features $\mathcal{F}$, we define the set of feature terms $\mathcal{T}$ and categories $\mathcal{C}$ as follows, where ' $\%$ ' and ' $Y$ ' are the standard LCG forward and backward implication operators.

$$
\begin{aligned}
& \mathcal{T}::=\mathcal{F}+\mathcal{T} \wedge \mathcal{T}+\mathcal{T} \vee \mathcal{T} \\
& \mathcal{C}::=\mathcal{T}+\mathcal{C} / \mathcal{C}+\mathcal{C} \backslash \mathcal{C}
\end{aligned}
$$

In general, atomic categories in a standard categorial grammar will be replaced in our analyses with formulae drawn from $\mathcal{T}$. For example, the NP $\mathrm{Kim}$ might be assigned by the lexicon to the category $n p \wedge s g \wedge 3$, the verb sleeps to the category $s \backslash n p \wedge s g \wedge 3$, 
and the verb slept (which does not impose person or number features on its subject) to the category $s \backslash n p$.

To simplify the presentation of the proofs, we formulate our system in natural deduction terms, and specify the properties of the Boolean connectives using the single inference rule $P$, rather than providing separate rules for each connective.

$\frac{\phi}{\psi} P$ where $\phi \vdash \psi$ in the propositional calculus. ${ }^{1}$

The rule $P$ allows us to replace any formula in $\mathcal{T}$ with a logically weaker one. For example, since Kim is assigned to the category $n p \wedge s g \wedge 3$, then by rule $P$ it will belong to $n p$ as well.

Finally, we assume the standard LCG introduction and elimination rules for the directed implication operators.

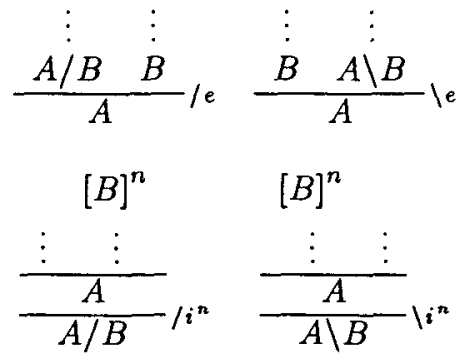

For example, the following proof of the wellformedness of the sentence Kim slept can be derived using the rules just given and the lexical assignments described above.

$\frac{\frac{\operatorname{Kim}}{n p \wedge s g \wedge 3}-\frac{\text { slept }}{s \backslash n p}}{s} \backslash e$

This example brings out one of the fundamental differences between the standard treatment of agreement in 'unification-based' grammar and this treatment of agreement in LCG. In the 'unification-based' accounts agreement is generally a symmetric relationship between the agreeing constituents: both agreeing constituents impose constraints on a shared agreement value, and the construction is well-formed iff these constraints are consistent.

However, in the LCG treatment of agreement proposed here agreement is inherently asymmetric, in

\footnotetext{
${ }^{1}$ Because conjunction and disjunction are the only connectives we permit, it does not matter whether we use the classical or intuitionistic propositional calculus here. In fact, if categories such as $n p$ and $a p$ are 'decomposed' into the conjunctions of atomic features

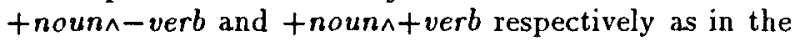
Sag et. al. (1985) analysis discussed below, disjunction is not required in any of the LCG analyses below. However, Bayer (1994) argues that such a decomposition is not always plausible.
}

that an argument must logically imply, or be $s u b$ sumed by, the antecedent of the predicate it combines with. Thus in the example above, the rule $P$ could be used to 'weaken' the argument from $n p \wedge s g \wedge 3$ to $n p$, but it would not allow $n p$ (without agreement features) to be 'strengthened' to, say, $n p \wedge s g \wedge 3$.

Abstracting from the details of the feature systems, we can characterize the 'unification-based' approach as one in which agreement is possible between two constituents with feature specifications $\phi$ and $\psi$ iff $\phi$ and $\psi$ are consistent, whereas the LCG approach requires that the argument $\phi$ implies the corresponding antecedent $\psi$ of the predicate (i.e., $\phi \vDash \psi)$.

Interestingly, in cases where features are fully specified, these subsumption and consistency requirements are equivalent. More precisely, say that a formula $\phi$ from a feature constraint language fixes an atomic feature constraint $\chi$ iff $\phi \vDash \chi$ or $\phi \vDash$ $\neg \chi$. For example, in single-valued feature systems $\langle$ person $\rangle=1$ and $\langle$ person $\rangle=3$ both $\operatorname{fix}\langle$ person $\rangle=1$, $\langle$ person $\rangle=2$, $\langle$ person $\rangle=3$, etc., and in general all fully-specified agreement constraints fix the same set of formulae.

Now let $\phi$ and $\psi$ be two satisfiable formulae that fix the same set of atomic feature constraints. Then $\phi \wedge \psi$ is consistent iff $\phi \models \psi$. To see this, note that because $\phi$ and $\psi$ fix the same set of formulae, each condition holds iff $\phi$ and $\psi$ are elementarily equivalent (i.e., for each feature constraint $\chi, \phi \vDash \chi$ iff $\psi \models \chi$ ).

However, the role of partial agreement feature specifications in the two systems is very different. The following sections explore the empirical consequences of these two approaches. We focus on coordination phenomena because this is the one area of the grammar where underspecified agreement features seem to play a crucial linguistic role, and cannot be regarded merely as an abbreviatory device for a disjunction of fully-specified agreement values.

\section{Coordination and agreement asymmetries}

Interestingly, the analysis of coordination is the one place where most 'unification-based' accounts abandon the symmetric consistency-based treatment of agreement and adopt an asymmetric subsumptionbased account. Working in the GPSG framework Sag et. al. (1985) proposed that the features on a conjunction must be the most specific category which subsumes each conjunct (called the generalization by Shieber (1992)). Shieber (1986) proposed a weaker condition, namely that the features on the conjunction must subsume the features on each conjunct, as expressed in the annotated phrase struc- 


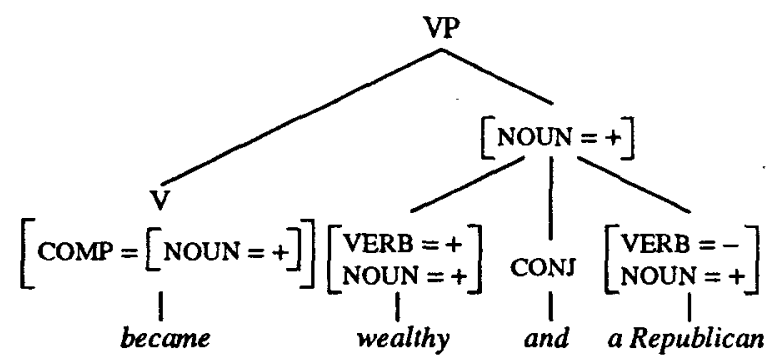

Figure 1: The feature structure subsumption analysis of (2b).

ture rule below (Shieber, 1992). ${ }^{2}$ In all of the examples we discuss below, the features associated with a conjunction is the generalization of the features associated with each of its conjuncts, so our conclusions are equally valid for both the generalization and subsumption accounts of coordination.

$$
\begin{aligned}
& X_{0} \longrightarrow X_{1} \operatorname{conj} X_{2} \\
& \quad \text { where } X_{0} \sqsubseteq X_{1} \text { and } X_{0} \sqsubseteq X_{2}
\end{aligned}
$$

Consider the sentences in (2). Decomposing the categories $\mathrm{N}$ (oun) and $\mathrm{A}$ (djective) into the Booleanvalued features $\{($ noun $\rangle=+,\langle$ verb $\rangle=-\}$ and $\{\langle$ noun $\rangle=+,\langle$ ver $b\rangle=+\}$ respectively, the fact that became can select for either an NP or an AP complement ( $2 \mathrm{a}$ ) can be captured by analysing it as subcategorizing for a complement whose category is underspecified; i.e., its complement satisfies $\langle$ noun $\rangle=+$, and no constraint is imposed on the verb feature.

(2) a. Kim [v became ] [AP wealthy ] / [NP a Republican ]

b. Kim [VP [v became] [AP wealthy] and [NP a Republican ] ]

Now consider the coordination in (2b). Assuming that became selects the underspecified category $\langle$ noun $\rangle=+$, the features associated with the coordination subsume the features associated with each coordinate, as required by rule (1), so (2b) has the well-formed structure shown in Figure 1.

On the other hand, a verb such as grew which selects solely AP complements (3a) requires that its complement satisfies $\langle$ noun $\rangle=+,\langle$ ver $b\rangle=+$. Thus the features on the coordinate structure in (3b) must include $\langle$ ver $b\rangle=+$ and so do not subsume the $\langle$ verb $\rangle=-$ feature on the NP complement, correctly predicting the ungrammaticality of $(3 \mathrm{~b})$.

(3) a. Kim grew [AP wealthy]/*[NP a Republican]

\footnotetext{
${ }^{2}$ Note that the LFG account of coordination provided by Kaplan and Maxwell (1988) differs significantly from both the generalization and the subsumption accounts of coordination just mentioned, and does not generate the incorrect predictions described below.
}

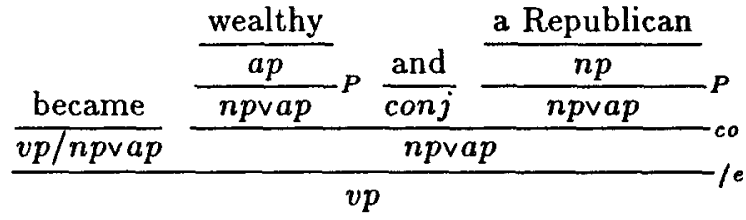

Figure 2: The LCG analysis of (2b).

$$
\frac{\text { grew }}{v p / a p} \frac{\frac{\text { wealthy }}{a p}}{\frac{n p \vee a p}{\text { n }} P \frac{\text { and }}{\operatorname{conj}} \frac{\frac{\text { a Republican }}{n p}}{n p \vee a p}}+P
$$

Figure 3: A blocked LCG analysis of the ungrammatical (3b)

b. ${ }^{*}$ Kim [vP [v grew ] [AP wealthy ] and [NP a Republican ] ]

Our LCG account analyses these constructions in a similar way. Because the LCG account of agreement has subsumption 'built in', the coordination rule merely requires identity of the conjunction and each of the conjuncts.

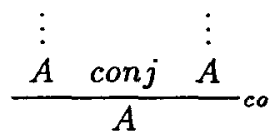

Condition: No undischarged assumptions in any conjunct. ${ }^{3}$

We provide an LCG derivation of (2b) in Figure 2. Roughly speaking, rule $P$ allows both the AP wealthy and the NP a Republican to 'weaken' to $n p \vee a p$, so the conjunction satisfies the antecedent of the predicate became. (This weakening also takes place in non-coordination examples such as Kim became wealthy). On the other hand, (3b) is correctly predicted to be ill-formed because the strongest possible category for the coordination is npvap, but this does not imply the 'stronger' $a p$ antecedent of grew, so the derivation in Figure 3 cannot proceed to form a $v p$.

Thus on these examples, the feature-based subsumption account and the LCG of complement coordination constructions impose similiar feature constraints; they both require that the predicate's feature specification of the complement subsumes the features of each of the arguments. In the featurebased account, this is because the features associated with a conjunction must subsume the features

\footnotetext{
${ }^{3}$ This condition in effect makes conjunctions into islands. Morrill (1992) shows how such island constraints can be expressed using modal extensions to LCG.
} 
associated with each conjunct, while in the LCG account the features associated with the complement specification in a predicate must subsume those associated with the complement itself.

Now consider the related construction in (4) involving conjoined predicates as well conjoined arguments. Similar constructions, and their relevance to the GPSG treatment of coordination, were first discussed by Jacobson (1987). In such cases, the feature-based subsumption account requires that the features associated with the predicate conjunction subsume those associated with each predicate conjunct. This is possible, as shown in Figure 4. Thus the feature structure subsumption account incorrectly predicts the well-formedness of (4).

(4) ${ }^{*} \mathrm{Kim}$ [ grew and remained ] [ wealthy and a Republican ].

Because the subsumption constraint in the LCG analysis is associated with the predicate-argument relationship (rather than the coordination construction, as in the feature-based subsumption account), an LCG analysis paralleling the one given in Figure 4 does not exist. By introducing and withdrawing a hypothetical ap constituent as shown in Figure 5 it is possible to conjoin grew and remained, but the resulting conjunction belongs to the category $v p / a p$, and cannot combine with the wealthy and a Republican, which belongs to the category npvap.

Informally, while rule $P$ allows the features associated with an argument to be weakened, together with the introduction and elimination rules it permits the argument specifications of predicates to be strengthened (c.f. the subproof showing that remained belongs to category $v p / a p$ in Figure 5). As we remarked earlier, in LCG predicates are analysed as (directed) implicational formulae, and the argument features required by a predicate appear in the $a n$ tecedent of such formulae. Since strengthening the antecedent of an implication weakens the implication as a whole, the combined effect of rule $P$ and the introduction and elimination rules is to permit the overall weakening of a category.

\section{Consistency and agreement}

Complex feature structure analyses of agreement require that certain combinations of feature constraints are inconsistent in order to correctly reflect agreement failure. For example, the agreement failure in him runs is reflected in the inconsistency of the constraints $\langle$ case $\rangle=a c c$ and $\langle$ case $\rangle=$ nom. In the LCG account presented above, the agreement failure in him runs is reflected by the failure of acc to imply nom, not by the inconsistency of the features acc and nom. Thus in LCG there is no principled reason not to assign a category an apparently contradictory feature specification such as $n p \wedge n o m \wedge a c c$ (this might be a reasonable lexical category assignment for an NP such as Kim).

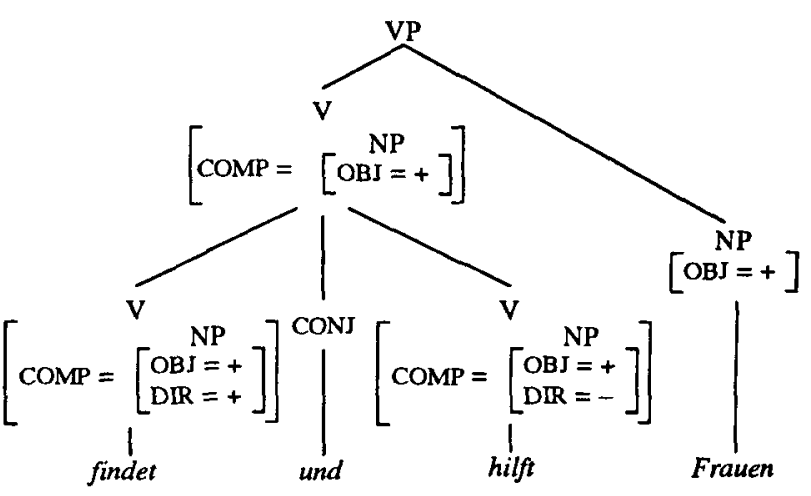

Figure 6: The feature structure subsumption analysis of $(5 c)$.

Consider the German examples in (5), cited by Pullum and Zwicky (1986) and Ingria (1990). These examples show that while the conjunction findet und hilft cannot take either a purely accusative (5a) or dative complement (5b), it can combine with the NP Frauen (5c), which can appear in both accusative and dative contexts.

(5)

$$
\begin{aligned}
& \text { a. * Er findet und hilft Männer } \\
& \text { he find-ACC and help-DAT men-ACC } \\
& \text { b. * Er findet und hilft Kindern } \\
& \text { he find-ACC and help-DAT children-DAT } \\
& \text { c. Er findet find-ACC and hilft } \\
& \text { he help-DAT } \\
& \text { women-ACC+DAT }
\end{aligned}
$$

Contrary to the claim by Ingria (1990), these examples can be accounted for straight-forwardly using the standard feature subsumption-based account of coordination. Now, this account presupposes the existence of appropriate underspecified categories (e.g., in the English example above it was crucial that major category labels were decomposed into the features noun and verb). Similarly, we decompose the four nominal cases in German into the 'subcase' features obj (abbreviating 'objective') and dir (for 'direct') as follows.

$$
\begin{array}{ll}
\text { Nominative } & \{\langle\text { dir }\rangle=+,\langle o b j\rangle=-\} \\
\text { Accusative } & \{\langle\text { dir }\rangle=+,\langle o b j\rangle=+\} \\
\text { Dative } & \{\langle\text { dir }\rangle=-,\langle o b j\rangle=+\} \\
\text { Gent-tive } & \{\langle\text { dir }\rangle=-,\langle o b j\rangle=-\}
\end{array}
$$

By assigning the NPs Männer and Kindern the fully specified case features shown above, and Frauen the underspecified case feature $\langle o b j\rangle=+$, both the feature structure generalization and subsumption accounts of coordination fail to generate the ungrammatical (5a) and (5b), and correctly accept (5c), as shown in Figure 6. 


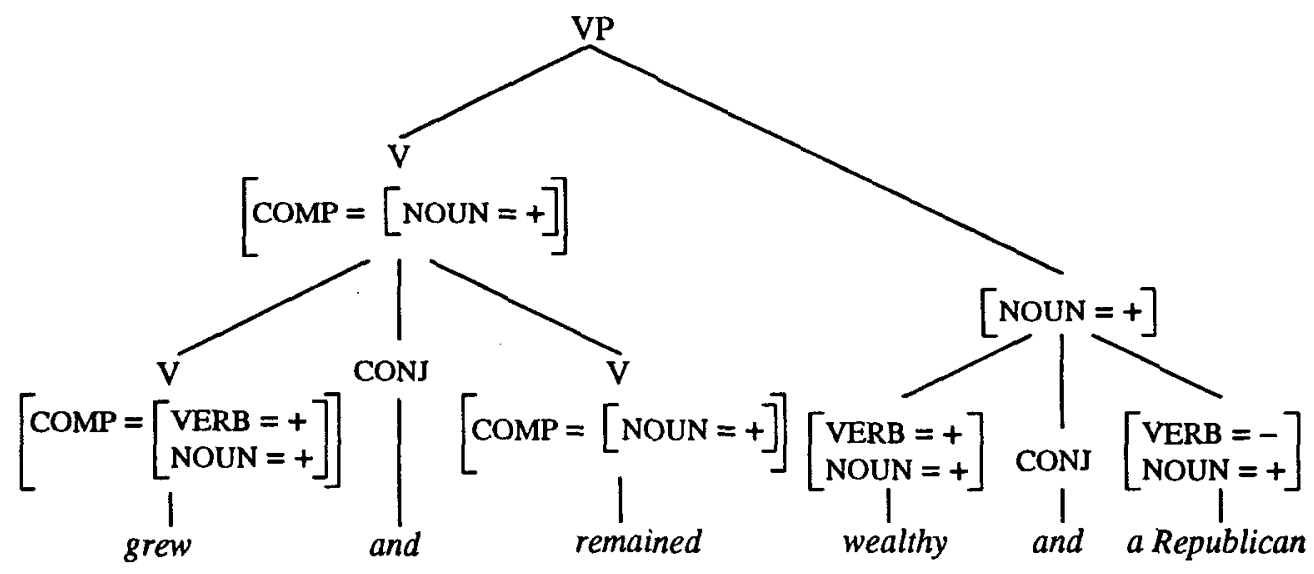

Figure 4: The feature structure subsumption analysis of the ungrammatical (4).

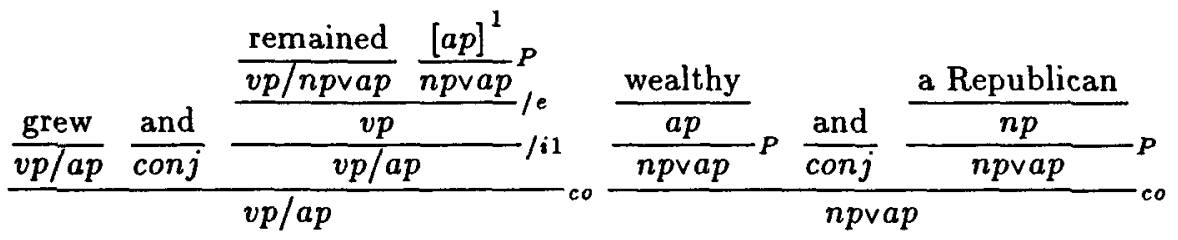

Figure 5: A blocked LCG analysis of the ungrammatical (4).

As in the previous example, the LCG approach does not require the case feature to be decomposed. However, as shown in Figure 7 it does assign the conjunction findet und hilft to the category $v p / n p \wedge a c c \wedge d a t$; hence the analysis requires that Frauen be assigned to the 'inconsistent' category $n p \wedge a c c \wedge d a t$. Such overspecified or 'inconsistent' features may seem ad hoc and unmotivated, but they arise naturally in the formal framework of Morrill's extended LCG.

In fact, they seem to be necessary to obtain a linguistically correct description of coordination in German. Consider the ungrammatical 'double coordination' example in (6). Both the feature structure generalization and subsumption accounts incorrectly predict it to be well-formed, as shown in Figure 8.

(6) * Er findet und hilft Männer und he find-ACC and help-DAT men-ACC and Kindern children-DAT

However, the LCG analysis systematically distinguishes between Frauen, which is assigned to the category $n p \wedge a c c \wedge d a t$, and Männer und Kindern, which is assigned to the weaker category $n p \wedge($ acc $\vee d a t)$. Thus the LCG analysis correctly predicts (6) to be ungrammatical, as shown in Figure 9. The

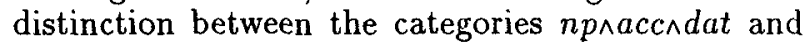
$n p \wedge($ acc $d a t)$, and hence the existence of the appar- ently inconsistent categories, seems to be crucial to the ability to distinguish between the grammatical (5c) and the ungrammatical (6).

\section{Conclusion}

This paper has examined some of the differences between a standard complex feature-structure account of agreement, which is fundamentally organized around a notion of consistency, and an account in an extended version of LCG, in which agreement is fundamentally an asymmetric relationship. We have attempted to show that the LCG account of agreement correctly treats a number of cases of coordination which are problematic for the standard feature-based account. Although we have not shown this here, the LCG account extends straightforwardly to the cases of coordination and morphological neutralization discussed by Zaenen and Kartunen (1984), Pullum and Zwicky (1986) and Ingria (1990).

The nature of an appropriate feature system for LCG is still an open question. It is perhaps surprising that the simple feature system proposed here can handle such complex linguistic phenomena, but additional mechanisms might be required to treat other linguistic constructions. The standard account of adverbial modification in standard LCG, for instance, treat; adverbs as functors. Because the verb 


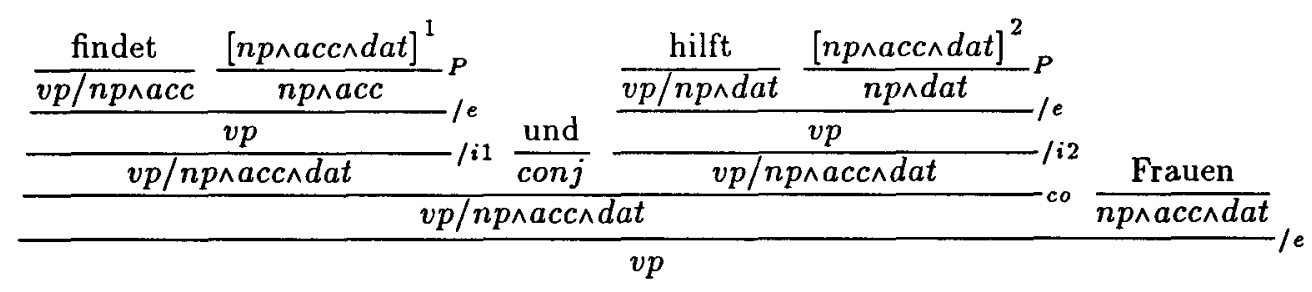

Figure 7: The LCG analysis of (5c)

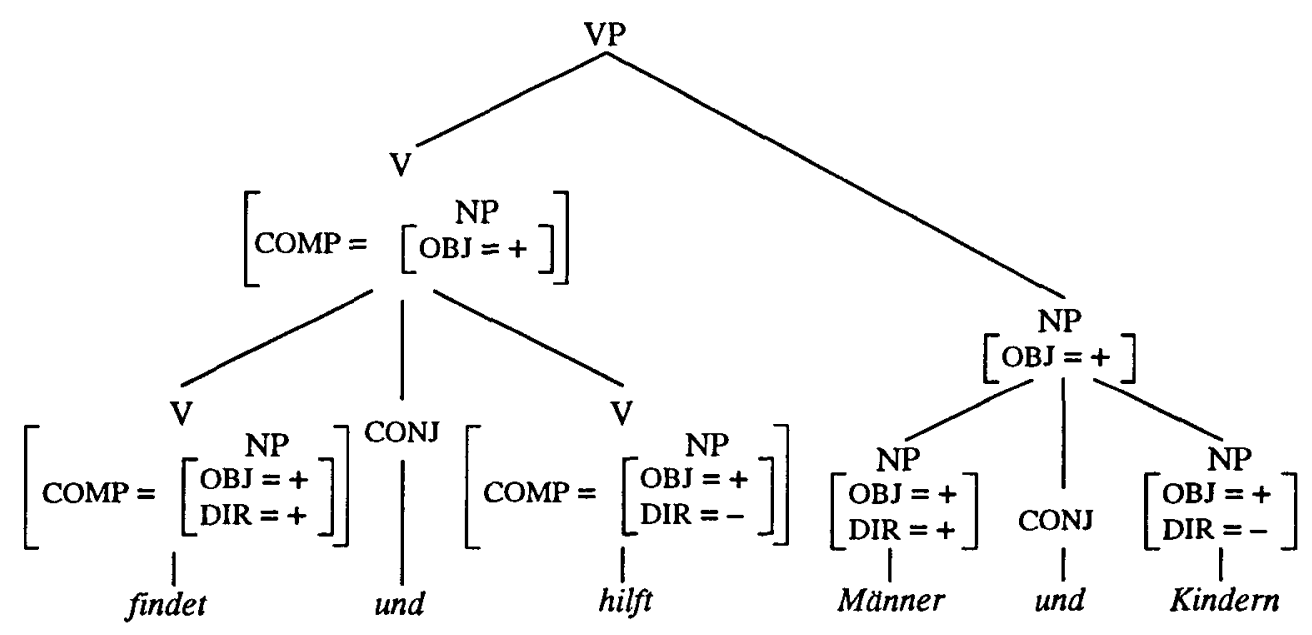

Figure 8: The feature structure subsumption analysis of the ungrammatical (6).

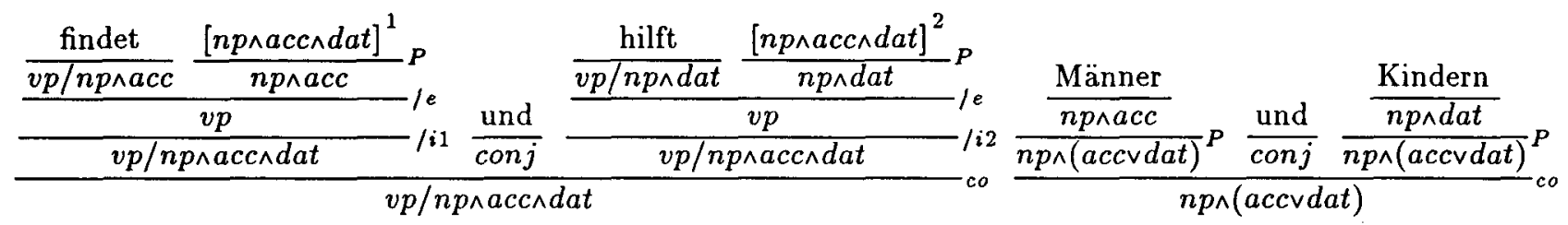

Figure 9: The blocked LCG analysis of the ungrammatical (6) 
heading an adverbial modified VP agrees in number with its subject, the same number features will have to appear in both the antecedent and consequent of the adverb. Using the LCG account described above it is necessary to treat adverbs as ambiguous, assigning them to the categories $(s \backslash n p \wedge s g) \backslash(s \backslash n p \wedge s g)$ and $(s \backslash n p \wedge p l) \backslash(s \backslash n p \wedge p l)$.

There are several approaches which may eliminate the need for such systematic ambiguity. First, if the language of (category) types is extended to permit universally quantified types as suggested by Morrill (Morrill, 1992), then adverbs could be assigned to the single type

$$
\forall X .((s \backslash n p \wedge X) \backslash(s \backslash n p \wedge X)) .
$$

Second, it might be possible to reanalyse adjunction in such a way that avoids the problem altogether. For example, Bouma and van Noord (1994) show that assuming that heads subcategorize for adjuncts (rather than the other way around, as is standard) permits a particularly elegant account of the double infinitive construction in Dutch. If adjuncts in general are treated as arguments of the head, then the 'problem' of 'passing features' through adjunction disappears.

The comparative computational complexity of both the unification-based approach and the LCG accounts is also of interest. Despite their simplicity, the computational complexity of the kinds of feature-structure and LCG grammars discussed here is largely unknown. Dorre et. al. (1992) showed that the satisfiability problem for systems of featurestructure subsumption and equality constraints is undecidable, but it is not clear if such problems can arise in the kinds of feature-structure grammars discussed above. Conversely, while terminating (Gentzen) proof procedures are available for extended LCG systems of the kind we presented here, none of these handle the coordination schema, and as far as we are aware the computational properties of systems which include this schema are largely unexplored.

\section{References}

Samuel Bayer. 1994. The coordination of unlike categories. Cognitive and Linguistic Sciences, Brown University.

Gosse Bouma and Gertjan van Noord. 1994. Constraint-based categorial grammar. In The Proceedings of the 32nd Annual Meeting of the Association for Computational Linguistics, pages 147-154, New Mexico State University - Las Cruces.

Jochen Dörre and William C. Rounds. 1992. On subsumption and semiunification in feature algebras. Journal of Symbolic Computation, 13:441461.
Jochen Dörre, Dov Gabbay, and Esther König. 1994. Fibred semantics for feature-based grammar logic. Technical report, Institute for Computational Linguistics, The University of Stuttgart.

Robert J. P. Ingria. 1990. The limits of unification. In The Proceedings of the 28th Annual Meeting of the Association for Computational Linguistics, pages 194-204, University of Pittsburgh.

Pauline Jacobson. 1987. Review of generalized phrase structure grammar. Linguistics and Philosophy, 10(3):389-426.

Ronald Kaplan and John T. Maxwell. 1988. Constituent coordination in lexical functional grammar. In The Proceedings of the 12th International Conference on Computational Linguistics, page 297302.

Joachim Lambek. 1958. The mathematics of sentence structure. American Mathematical Monthly, 65:154-170.

Anne-Marie Mineur. 1993. Disjunctive gender features-a comparison between HPSG and CG. DFKI, Saarbrücken.

Glyn V. Morrill. 1992. Type-logical grammar. Technical Report Report LSI-92-5-R, Departament de Llenguatges i sistemes informàtics.

Carl Pollard and Ivan Sag. 1994. Head-driven Phrase Structure Grammar. The University of Chicago Press, Chicago.

Geoffrey K. Pullum and Arnold M. Zwicky. 1986. Phonological resolution of syntactic feature conflict. Language, 62(4):751-773.

Ivan A. Sag, Gerald Gazdar, Thomas Wasow, and Steven Weisler. 1985. Coordination and how to distinguish categories. Natural Language and Linguistic Theory, 3(2):117-171.

Stuart M. Shieber. 1986. An Introduction to Unification-based Approaches to Grammar. CSLI Lecture Notes Series, The University of Chicago Press, Chicago.

Stuart M. Shieber. 1992. Constraint-based Grammar Formalisms. The MIT Press, Cambridge, Massachusetts.

Annie Zaenen and Lauri Karttunen. 1984. Morphological non-distinctiveness and coordination. In Proceedings of the Eastern States Conference on Linguistic3, volume 1, pages 309-320. 